\title{
Glaucoma, Stem Cells, and Gene Therapy: Where Are We Now?
}

\author{
Karim Daliri $^{1,2}$, Alexander V. Ljubimov ${ }^{3,4}$, Seyedhossein Hekmatimoghaddam ${ }^{5,6}$ \\ ${ }^{I}$ Neurogenetic Ward, Comprehensive Child Developmental Center, Shiraz University of Medical Sciences, Shiraz, Iran \\ ${ }^{2}$ Visiting Scientist at Department of Neurology and Epileptology, Hertie Institute for Clinical Brain Research, University of Tübingen, Germany \\ ${ }^{3}$ Regenerative Medicine Institute, Department of Biomedical Sciences, Cedars-Sinai Medical Center, Los Angeles, CA, USA \\ ${ }^{4}$ Department of Medicine, David Geffen School of Medicine at UCLA, Los Angeles, CA, USA \\ ${ }^{5}$ Stem Cell Biology Research Center, Yazd Reproductive Sciences Institute, Shahid Sadoughi University of Medical Sciences, Yazd, Iran \\ ${ }^{6}$ Department of Laboratory Sciences, School of Paramedicine, Shahid Sadoughi University of Medical Sciences, Yazd, Iran
}

Glaucoma is the second most common cause of blindness, affecting 70 80 million people around the world. The death of retinal ganglion cells (RGCs) is the main cause of blindness related to this disease. Current therapies do not provide enough protection and regeneration of RGCs. A novel opportunity for treatment of glaucoma is application of technologies related to stem cell and gene therapy. In this perspective we will thus focus on emerging approaches to glaucoma treatment including stem cells and gene therapy.

Keywords: Ganglion cells, Gene therapy, Glaucoma, Retina, Stem cells

\section{Introduction}

Glaucoma, the second most common cause of irreversible blindness in the world, is becoming increasingly prevalent as life expectancy increases. Currently, there is no permanent treatment that reverses the glaucomatous optic neuropathy that characterizes this disease (1).

The main cause of blindness related to glaucoma is the death of retinal ganglion cells (RGCs). Regeneration of lost RGCs is currently not feasible; therefore, prevention of the disease is the best approach in glaucoma (2).

Accepted for publication May 20, 2017, Published online November 30, 2017

Correspondence to Seyedhossein Hekmatimoghaddam

Department of Laboratory Sciences, School of Paramedicine, Emam Reza Complex, Daneshjoo Blvd, Yazd 8916188635, Iran

Tel: +98-9133518314, Fax: +98-3537246541

E-mail: shhekmati2002@yahoo.com

(c) This is an open-access article distributed under the terms of the Creative Commons Attribution Non-Commercial License (http://creativecommons.org/ licenses/by-nc/4.0/), which permits unrestricted non-commercial use, distribution, and reproduction in any medium, provided the original work is properly cited.

Copyright (c) 2017 by the Korean Society for Stem Cells Research
The most important feature of glaucoma is increased intraocular pressure (IOP). The major protocols for the treatment of glaucoma, whether pharmaceutical or surgical, have focused on the trabecular meshwork (TM), which is located around the base of the cornea and forms the outflow system ensuring drainage of the aqueous humor. TM therapy attempts to relieve the IOP and slow the progression of glaucoma (3). However, traditional TM-directed therapies are largely ineffective, and a number of approaches for recovering sight to the blind are being investigated, including prosthetic devices, stem cell transplants and gene therapy, which have shown various degrees of success. The former relies mainly on invasive surgeries and delivery of foreign material into the eye (4).

A new approach for optic nerve regeneration is the use of Müller glia that reportedly have some stem cell characteristics. These cells are well studied in lower vertebrates (e.g. amphibians, fish) and also in the adult human retina where they significantly contribute to the retinal structure, homeostasis, and regeneration. For example, Müller glial cells acquire stem cell characteristics with retinal regeneration capacity in zebrafish after injury to the retina (4-6).

Gene therapy is an experimental technique for treating 
disease thorough altering the patient's genetic material transiently or permanently. Gene therapy works by introducing a healthy copy of a defective coding or non-coding nucleic acid such as DNA, mRNA, small interfering RNA (siRNA), microRNA (miRNA), or antisense oligonucleotides into the patient's cells. Moreover, genome editing techniques, such as CRISPR/Cas9 have opened new avenues in the directional genome modifications by removing, replacing, or adding parts of the target DNA sequence. Gene transfer has shown promise for various eye diseases (5-7). However, there is a remarkable paucity of gene therapy trials for glaucoma.

\section{Cell-based therapies in human glaucoma}

A novel opportunity for the treatment of glaucoma with cell-based therapies is the application of Müller glia that may circumvent many concerns regarding the use of embryonic and pluripotent cells. Differentiation of Müller glia to retinal rod photoreceptors after injury (with the aid of growth factors and contribution of the Notch pathway) in lower mammals suggests neurogenic capability of Müller glia in regenerative medicine (8).

A new in vitro protocol to differentiate human Müller glia (considered a type of human mesenchymal stem cells, hMSCs) to rod photoreceptors with examination of the outcome by immunohistochemical analysis of cellular migration in ex vivo transplanted retina has recently introduced a new option in the long way of glial application in regenerative medicine. Recombinant human basic fibroblast growth factor (FGF-2), taurine, retinoic acid and insulin-like growth factor type 1 (IGF-1) are key factors of this protocol that has shown success in inducing morphological changes and rod photoreceptor gene expression in human Müller glia (9).

\section{Vascular endothelial growth factor (VEGF)-based therapy and Müller cells in glaucoma}

VEGF is an important angiogenic, vascular permeability, and neurotrophic factor. It plays a significant role in protein expression and peroxynitration, which are involved in retinal inflammation, neovascularization, vascular leakage, and other key pathological changes (10).

Hypoxia may be important for the development of pathological neovascularization leading to vision loss. Physiologically regulated gene expression change by hypoxia offers a great potential for more controlled gene therapy in ischemic visual disorders. A number of hypoxia-sensitive gene switches have been developed to target genes in hypoxic cells, which are based on hypoxia-response elements (HRE) (11).

HREs are targeted by hypoxia inducible factor (HIF), a heterodimer of HIF-1 $\alpha$ and HIF-1 $\beta$. In normoxia, HIF-1 $\alpha$ is hydroxylated and rapidly degraded by the proteasome, whereas in hypoxic conditions HIF-1 $\alpha$ is stabilized and accumulates in the cytoplasm to make HIF-1 dimers that bind to the HREs in target genes. Integrating HREs in gene therapy makes it HIF-1 - regulated. Thus, the therapy will be activated only in retinal regions experiencing hypoxia or other pathological conditions such as HIF-1 activated angiogenesis $(11,12)$.

Müller cells experience hypoxic stress following capillary loss in diabetes, and express VEGF in oxygen-induced retinopathy. It is known that Müller cell-derived VEGF is a significant contributor to neovascularization in the retina. Therefore, anti-VEGF gene therapy targeting Müller cells may be an important addition to existing gene therapies for glaucoma providing means to selectively deliver anti-angiogenic factors to either the inner retina or outer retina/choroid $(13,14)$.

Endostatin has profound inhibitory effects on angiogenesis, and should be considered as a new target for suppressing ocular neovascularization. A cleavage product of collagen XVIII, endostatin inhibits endothelial cell proliferation, migration and survival. It also increases expression of anti-angiogenic factors, and inhibits levels of pro-angiogenic factors. After internalization of endostatin via endocytosis or by clathrin coated pits and its localization in the nucleus, reduction of VEGF expression occurs along with augmentation of anti-angiogenic pigment epithelium-derived factor (PEDF) expression, and competition with VEGF for binding to VEGF receptor (15).

\section{CYP1Bl gene: a novel target for stem cell- and gene-based therapy in glaucoma}

CYP1B1 is a dioxin-inducible enzyme and a member of the cytochrome P450 superfamily. It has a significant function in the in utero development of ocular structures, and its dysfunction can lead to ocular developmental defects.

The role of CYPlBl gene alterations in primary congenital glaucoma (PCG) has been known for about a decade. Recent evidence has shown the involvement of $C Y P 1 B 1$ mutations in some types of glaucoma and anterior segment disorders, suggesting a wider role for CYP1B1 in ocular physiology (16).

Mutations in CYPIBI have been shown to impact the development of the trabecular meshwork, acting to shape 
or degrade some endobiotic compounds in the process of development of this filtering structure. Thus, a novel and more direct approach would be to try correcting or replacing aberrant forms of CYP1B1. Furthermore, differentiating into a specific lineage and transferring stem cells containing wild-type $C Y P 1 B 1$ may be tried for particular regions of the eye where they will develop into normal cells of that region and correct the defect. This approach might be applicable in families with PCG and defined mutations in the CYP1B1 gene (17).

\section{Potential use of Atoh7 gene}

The differentiation of Müller cells to retinal stem cells or RGCs in certain conditions holds promise for regeneration of optic nerve defects in glaucoma. Atoh7 is a key transcription factor for RGC differentiation. Atoh7 mutant mice can survive and their optic nerve is observable but lacks RGCs. Knocking out Atoh7 in mouse embryonic stem cells blocks the differentiation of about $80 \%$ of RGCs (18).

Moreover, the ATOH7 gene has been previously shown to be related to glaucoma and glaucoma-related traits, such as disc size and cup/disc ratio (CDR). CDR is a main parameter of the glaucoma phenotype (19). Atoh7 overexpression may increase the differentiation of cultured retinal stem cells to RGCs. Also, Müller glial cells transfected with the PEGFP-N1-Atoh7 plasmid could be differentiated into large quantities of RGCs $(20,21)$.

\section{Main tools in glaucoma-based gene therapy}

As gene therapy vehicles, healthy Müller cells could be modified to constitutively secrete a broad range of neurotrophic factors (NTFs) such as GDNF and FGF or other factors, under universal or cell-specific promoters or even under inducible promoters. Of the latter, the stress-inducible promoters of glial fibrillary acidic protein (GFAP), vimentin, and CD44, for instance, have been suggested as potential options in developing novel DNA-based therapeutic tools to target glaucoma.

In general, a strong promoter is a critical element in gene therapy, but in practice where limited expression is desired such as targeting transcription to a specific tissue or cell, a toolbox of particular promoters would be advantageous. For example, human paired box 6 MiniPromoters (PAX6 MiniPs) is a well-designed large promoter composed of seven small promoters for targeting therapeutically desired cells in the gene therapy of diseases influencing the inner retina such as diabetic retinopathy and glaucoma. Ple255 and Ple259 are two PAX6 MiniPs. They are particularly interesting in that each captures three of the four cell types expressing PAX6, and influences the entire PAX6 expression in mouse retina. Specificity and stability are special characteristics of a MiniP; therefore, both promoters are appealing choices for future optimization to impact the whole retinal expression pattern of PAX6, and for application in PAX6 gene therapy in ocular disorders (22).

\section{Cyclooxygenase-2 and reduction of IOP in glaucoma}

Cyclooxygenase-2 (COX-2) is a rate-limiting enzyme in prostaglandin (PG) biosynthesis. Loss of COX-2 expression in aqueous humor-secreting cells of the eye has been associated with primary open-angle glaucoma (POAG). Barraza et al. used lentiviral vectors for expressing COX2 and other PG biosynthesis genes in the ciliary body epithelium and TM. They showed that strong ectopic COX-2 expression and PG production require COX-2 cDNA sequence optimization. Additionally, coupling COX-2 expression with synthetic PGF2 $\alpha$ receptor transgene revealed notable and stable reductions in IOP in a domestic cat $(23,24)$. This is the first gene therapy study for correcting the main cause of glaucoma, which opens a new avenue for future research in glaucoma including control of IOP through anterior chamber injection of cells modified to secrete COX-2. It has also been suggested that IOP control might be augmented in some types of glaucoma through TM extracellular matrix modification (25).

\section{Potential of neurotrophic factors for cell-based gene therapy in glaucoma}

Neurotrophic factors are secreted proteins that play important roles in the synaptic and neuronal growth, pruning, myelination, differentiation, and survival of neurons. Two main neurotrophic factors will be addressed here.

\section{Brain-derived neurotrophic factor (BDNF)}

BDNF levels in the serum of patients with POAG and the tears of normotensive glaucoma patients are significantly lower than that of control subjects, suggesting that BDNF may be a biomarker for glaucoma. Virus-mediated gene expression of BDNF in the retina promotes robust RGC survival in various experimental glaucoma models, including optic nerve transection and high IOP (26). Interestingly, BDNF gene delivery to Müller glia also prolonged RGC survival, indicating an important role of glia 
in neuroprotection. Furthermore, overexpression of BDNF receptor TrkB in RGCs can stimulate RGC survival following optic nerve transection. These findings indicate that BDNF-TrkB signaling is another good therapeutic target for glaucoma (27). Indeed, increasing the phosphatidylinositol 3-kinase (PI3K)/Akt by TrkB deactivates pro-apoptotic elements and the extracellular signal-regulated kinase (ERK). Then, the expression of neuronal survival genes is activated by phosphorylation of the cAMP response element binding protein (CREB) (28). Furthermore, antibodies, which selectively activate $\operatorname{TrkB}$ can improve RGC survival in glaucoma animal models suggesting that activation of TrkB is a potential therapy for relieving glaucoma (29).

It has also has been proposed that augmenting the concentration of neurotrophic factors within ganglion cells will impede cell death seen in glaucoma (30).

Levkovitch-Verbin et al. have shown that MSCs can be induced to over-secrete BDNF and GDNF without direct genetic manipulation but through incubation in novel culture conditions. They reported significant neuroprotective benefits in RGCs after optic nerve transection. Co-expression of a LINGO-1 antagonist has been suggested to overcome transient effectiveness of BDNF (31).

\section{Glial cell line-derived neurotrophic factor (GDNF)}

GDNF belongs to the GDNF family, and is a distant member of the transforming growth factor (TGF) superfamily. GDNF was originally identified as a potent neurotrophic factor that enhances survival of midbrain dopaminergic neurons (32). In addition, GDNF has been reported to protect RGCs following optic nerve transection or retinal ischemia. In glaucoma model, DBA/2J mice, intravitreal injection of microspheres with encapsulated GDNF remarkably improved long-term RGC survival. GDNF appears to be promising as a potentially strong therapeutic modality for retinal diseases, especially for retinitis pigmentosa and glaucoma $(32,33)$. Overexpression of the glutamate/aspartate transporter (GLAST) in Müller glia by GDNF is critical for protecting RGC, suggesting that GLAST may be another target of gene therapy in glaucoma (34).

\section{IGF-1}

IGF-1 is produced by many cell types in response to growth hormone secretion by the pituitary gland, and is regarded as an essential factor for the regulation of cell growth and central nervous system development. IGF-1 and its receptor (IGF-1R) system are able to stimulate growth in many different cell types and prevent apoptosis.
In addition, IGF-1 can significantly promote proliferation, survival of neurons, mitosis in sympathetic neuroblasts, and neurite outgrowth in motor neurons (35). Applying human neuronal progenitor cells (hNPC) transfected with vectors carrying IGF-TD (td Tomato) to deliver active IGF-1 fusion protein in a rodent has been reported to suppress RGC loss in experimental glaucoma (36).

\section{CRB1 and CRB2 as potential therapeutic markers in retinal dystrophies}

Human Crumbs-homologue-1 (CRBI) gene is expressed above the adherens junctions in Müller cells and in the inner segments of photoreceptors, whereas CRB2 is localized only in Müller cells. Mutations in the $C R B 1$ gene cause severe autosomal recessive retinal dystrophies and chronic vision disorders in about 80,000 patients worldwide (37). In mice, however, Crbl is expressed only in Müller glial cells whereas Crb2 is expressed in both photoreceptors and Müller cells. The products of both genes have similar functions including the lamination of the retina, maintenance of the adherens junctions between photoreceptors and Müller cells, restraining the number of progenitor cells and controlling Notch and Hippo signaling pathways. Human $C R B 2$ has high similarity in homology and function with mouse $C R B 2$ but has smaller size. Using the human $C R B 2$ cDNA AAV transduction CRB2 protein was expressed and located correctly at the subapical area of mouse retina. Rescue experiments with $C R B 1$ and $C R B 2$ vectors in severely disorganized retina of $\mathrm{CrblCrb2}{ }^{F /+} \mathrm{cKO}$ and $\mathrm{Crb} 2 \mathrm{cKO}$ mouse model showed that only CRB2 targeted in both Müller cells and photoreceptors prevented further loss of retinal function in these models. Interestingly, $C R B I$ had adverse effects on retinal activity if retina lacks endogenous Crbl, whereas over-expression of $C R B 1$ or $C R B 2$ in retinas expressing endogenous mouse $\mathrm{Crb} 1$ and $\mathrm{Crb} 2$ did not have these effects $(38,39)$.

\section{siRNA-based strategy for glaucoma}

Small interfering RNAs (siRNAs) are double-stranded RNA molecules 20 25 nucleotides long that regulate gene expression by targeting messenger RNA to degradation leading to gene silencing. siRNAs are a common therapeutic method for silencing the expression of deleterious genes in an extensive range of disorders (40). Here, siRNA application in glaucoma studies is briefly reviewed.

A synthetic siRNA, QPI-1007, that blocks the expression of caspase 2 is being developed as a neuro- 
protective treatment in glaucoma. Yu-Wai-Man et al. developed an effective delivery system containing receptor-targeted liposome-peptide-siRNA nanoparticles for MRTF-B siRNAs in human Tenon's fibroblasts, which impeded post-surgical fibrosis after glaucoma filtration surgery (41). In enriched primary RGC cultures, the effect on Sncg expression was studied after siRNA-based knockdown of PFDN2 gene. Gene expression analyses revealed effective downregulation of Sncg in RGCs treated with $\operatorname{Pfdn} 2$ siRNA, demonstrating that Pfdn2 is upstream of Sncg and directly modulates Sncg expression (42).

Recent studies suggest that neuronal loss in Alzheimer's disease (AD) and glaucoma is mediated by common neurodegenerative pathways. For instance, aggregations of amyloid $\beta(\mathrm{A} \beta)$ peptides have been shown in experimental glaucoma, and blocking their accumulation decreases RGC loss. Additionally, it is recognized that tau is a key mediator of $\mathrm{A} \beta$ peptide toxicity. Transgenic mice with the human P301S tau mutant contain tau aggregates in RGCs, suggesting an association between tau accumulation and RGC dysfunction. Using siRNA as a novel therapy for glaucomatous eyes through injection into the vitreous cavity in rats to target tau protein showed rapid uptake by RGCs, and successfully reduced retinal tau levels, leading to the improvement of RGC body and axon survival (43). Furthermore, changing the pattern of tau phosphorylation may be a potential approach in preventing formation of tau oligomers and RGC loss. Thus, by blocking retinal tau protein available for phosphorylation, siTau can probably reduce tau accumulation and neuronal death (43).

Using siRNA to investigate the molecular pathways of eye disorders is another application of siRNAs. For example, knocking down Smad4 or $\beta$-catenin (which are part of TGF- $\beta$ and Wnt signaling pathways) by siRNA was investigated. The existence of a cross-inhibition between the TGF $\beta /$ Smad and canonical Wnt pathways in the TM was shown. It may be important for future therapy, as the Wnt pathway inhibitor and TGF- $\beta$ signaling pathway activator, secreted frizzled-related protein 1 (SFRP1), is increased in POAG $(44,45)$.

\section{Mitochondrial-based gene therapy in glaucoma}

Mutations of mtDNA or nDNA encoding mitochondrial proteins can lead to mitochondrial dysfunction. A growing body of evidence suggests that chronically elevated oxidative stress is a pathological feature for triggering neuronal death in glaucoma indicating the participation of mitochondria as a main endogenous source for reactive oxy- gen species (ROS) in accelerating the loss of RGCs. Indeed, in the anterior part of the human eye, oxidative stress has been linked to cataract and glaucoma, whereas in the posterior segment macular degeneration may occur. Moreover, oxidative stress can trigger epigenetic changes such as DNA methylation and chromatin remodeling, which may cause some clinical manifestations. Therefore, the antioxidant agents are assumed to have protective role against degenerative ocular pathologies $(46,47)$. For instance, a significant reduction of superoxide ion upon administration of AAV-SOD2 into the rat retina with increased expression of superoxide dismutase (SOD2) gene attenuated RGCs death and may be a promising glaucoma gene therapy approach $(48,49)$.

Another experimental strategy is based on the assumption that modifications of dynamin-related protein 1 (DRP1) are linked to mitochondrial dysfunction-mediated bioenergetic failure and neuronal cell apoptosis. Inhibition of DRP1 activity by overexpression of $\mathrm{DRP} 1^{\mathrm{K} 38 \mathrm{~A}}$ or by inhibitors like Mdivi-1 and P110-TAT blocks mitochondrial fission and cell death in neurodegenerative disorders (50). More recently, by using Mdivi-1, the promotion of RGC survival in the condition of IOP elevation was reported, which led to the assumption that DRP1 inhibition may rescue RGCs from mitochondrial fission-mediated oxidative stress in glaucoma. Increasing SOD2 and mitochondrial transcription factor A (Tfam) expression following overexpression of DRP1 triggered by elevated oxidative stress in the retina of glaucomatous D2 mouse model as well as in cultured RGCs may constitute a novel approach for mitochondrial-based gene therapies (46).

\section{Gene delivery approaches in glaucoma}

Unique anatomy and physiology of the eye makes the development of a less invasive retinal delivery system a challenge. However, the retina itself has some special advantages for gene therapy including easy accessibility for surgical injection, the small size of retina allowing to obtain a therapeutic effect with a low gene dose and the blood-retinal barrier conferring immune privilege to the retinal tissue and restricting systemic spread of the virus (51).

There are two general approaches for delivery of gene into cells, including nonviral and viral carriers. Nonviral-based approaches are often safer but may offer low transfection efficiency. Direct injection of a plasmid containing a target gene and using nanoparticles are two common types of nonviral-based approaches (52). Moloney murine leukemia virus (MoMLV), a serotype 5 adenovirus 
(Ad5), adeno-associated viruses (AAV) and lentiviruses (HIV-1-based vectors) are common viral vectors for transferring genes to target tissues. Viral vectors are efficient vehicles; however, some safety problems raise concerns about their use.

Nano delivery systems are powerful nonviral delivery vectors. Various ophthalmic applications of nanotechnology for several different retinal diseases focus on improved drug and gene delivery to target tissue in the retina. Nanodrugs offer the possibility of extensive modifications to improve their function and tissue targetability. For example, encapsulation of siRNA within a core with a layer of cationic polymer may facilitate cytoplasmic release. Covering the polymer with a coating such as polyethylene glycol may increase the cargo protection from degradation in vivo until its release into the cytoplasm. Functionalizing the nanocarriers with a specific targeting unit such as an antibody or aptamer for delivering the agent into a specific diseased tissue is also a promising way to increase gene therapy efficacy (53). Although some nanodrugs have been already tested for glaucoma treatment, there are no data yet of their use as gene therapy vehicles.

\section{Mesenchymal stem cells and glaucoma}

Multipotent bone marrow-derived mesenchymal stem cells (MSCs) have extensive potential for the delivery of therapeutic proteins to treat or study damaged organs. Transplantation of MSCs has attracted considerable attention in efforts to develop cell-based therapies because they are readily obtained from the patient. Encouraging results have been reported in animal models for various diseases, including spinal cord injury, stroke, and myelin deficiency. Another capacity of MSCs is the ability to migrate and differentiate into neural-like cells in vitro. Overall, cell-mediated neuroprotective and replacement functions appear to be a great advantage as a therapeutic modality in retinal degeneration and glaucoma $(54,55)$.

Engineering of stem cells for the production of neurotrophic growth factors has been proposed as an attractive model for delivery of neuroprotectants such as BDNF to the injured CNS, and its use for long-term treatment of glaucoma is a viable option. BDNF-MSC transplants can protect retinal and optic nerve function in animal models (56).

The most likely mechanism of the neuroprotective effect described by grafted MSCs is the secretion of neurotrophic growth factors. Delivery of neural stem cells can protect RGCs from glaucomatous death, but obstacles for obtaining and using neural stem cells are likely to impede their clinical applications. Other pluripotent stem cell sources, such as embryonic stem cells, have also been explored for their retinal neuroprotective qualities, but their usage is restricted by rejection and some ethical issues $(57,58)$.

Recent advances in MSC use are encouraging. Johanson et al. found that the MSCs survived for more than 35 days following intravitreal transplantation in a rat model of glaucoma, with notable migration of MSCs to the retina (59). MSCs originated from the trabecular meshwork and conjunctiva can generate photoreceptor-like cells in vitro as well (60). Furthermore, RGCs in models of transient ischemia showed improved survival upon bone marrow-derived stem cells (BMSCs) transplantation (61). In a successful clinical trial applying autologous BMSCs visual acuity of a 27-year old patient with idiopathic bilateral optic neuritis causing bilateral optic neuropathy was corrected significantly, and the correction remained stable one year after the operation. This could be due to various factors including secretion of neurotrophic factors such as $\mathrm{BDNF}$ or induction of GDNF and neurotrophin-3 production by BMSCs $(62,63)$. Taken together, the administration of autologous stem cell therapy with neuroprotective function utilizing effective local delivery is emerging as a novel and effective tool to treat eye disorders such as glaucoma.

\section{Using iPSCs in the treatment of glaucoma}

The advent of patient-specific induced pluripotent stem cells (iPSCs) has allowed scientists to access living diseased tissues that would have been unavailable for molecular studies. This novel technology has opened up three main areas of investigation, each of which is relevant to the treatment of inherited blindness: (a) analysis of specific mutations and their related pathophysiologic mechanisms; (b) assessment of new gene amplification, gene silencing, and other molecular therapies; and (c) recovery of the function by transplantation of new cells $(64,65)$.

The goal of iPSCs application is the restoration of vision in the patient in a specific location. These cells may be derived from the same tissue or from other tissues, such as skin. iPSCs may be corrected genetically by genome editing technologies such as CRISPR/Cas9; they are also immunologically suitable for regeneration of eye tissues. They are easily expandable and bankable. Using the patient's own cells, lifelong and expensive immunosuppression with significant side effects would not be necessary as well. However, the main obstacle of using iPSCs is technical difficulties including their generation, maintenance and differentiation, which are time-consum- 
ing, and need specialized equipment, protocols, and expertise $(66,67)$.

Around 200 various genes with thousands of different mutations have been reported to cause retinal degeneration. For example, mutations of OPTN and TBK1, MYOC genes can cause familial low-tension glaucoma that are directly associated with RGC death and lifelong loss of vision. Recently, iPSC-derived RGCs were generated from dermal fibroblasts of non glaucomatous individuals and from a patient with TBK1-associated low-tension glaucoma. Using these cells, it was demonstrated that duplication of the TBKI gene increases autophagy and augmentation of the lipidated form of microtubule-associated protein 1A/1B-light chain 3 (LC3) leading to cell death. Using patient-derived TBK1-deficient RGC, it could be possible to regulate autophagy and to bypass RGC death.

Transplantation of iPSC-TM into the eyes of Tg-MYO$\mathrm{CY} 437 \mathrm{H}$ mice prevented the development of elevated IOP and maintained aqueous humor normal outflow facility for at least nine weeks (68). Although these findings are very encouraging, a number of questions remain to be answered. For instance, as the functional restoration depends on proliferation of endogenous TM cells, which also express MYOCY437H, it is possible that these new cells will eventually be lost. Although in theory the eye can be retreated with iPSC-TM, the influence of old age is now unclear, and some approaches are less successful in eyes with very advanced disease. These considerations may be less of a concern in open angle glaucoma, which is not the result of a deleterious mutation. TM cell loss usually occurs over many decades with similar rates, and newly derived TM cells may sustain for many years. Indeed, more refined investigations will have to be performed to determine whether these methods can be successful in other animal models and eventually in patients.

\section{Emerging concepts and future perspectives}

Several promising approaches have been developed for the treatment of glaucoma and other ophthalmic neurodegenerative disorders using gene delivery, gene ablation, and stem cell transplantation. Each method has its own strengths and weaknesses, and selection of one over the other depends on the availability of necessary experience and resources. However, a number of important questions remain to be addressed in future studies including:

Among different studies on glaucoma stem cell based gene therapy, numerous investigations have been focused on the in vitro approach, and less on the in vivo models. In the future, more investigations need to be pursued in this area in animal models. Additionally, new models should be established in animals whose eyes are more similar to human, such as sheep, cow and chimpanzee.

Using autologous stem cells such as MSCs, BMSCs and iPSCs that have been genetically modified to have the upregulated/downregulated levels of genes having key roles in pathophysiology of glaucoma may constitute a promising future approach for treatment, although more preclinical studies are necessary.

Developing novel nanovectors as gene carriers with low risk of side effects, especially combinations of nanoparticles with antibodies or aptamers to target the specific cells may be another promising avenue of research in this area.

\section{Potential conflict of interest}

The authors have no conflicting financial interest.

\section{References}

1. Wang GQ, Bai ZX, Shi J, Luo S, Chang HF, Sai XY. Prevalence and risk factors for eye diseases, blindness, and low vision in Lhasa, Tibet. Int J Ophthalmol 2013;6:237-241

2. Xu ZR, Jiang FG, Chen F. Effects of abnormal optineurin expression on the survival of the rat retinal ganglion cell line RGC-5. Genet Mol Res 2015;14:9171-9180

3. Qiu X, Wu K, Lin X, Liu Q, Ye Y, Yu M. Dexamethasone increases Cdc42 expression in human TM-1 cells. Curr Eye Res 2015;40:290-299

4. Goldman D. Müller glial cell reprogramming and retina regeneration. Nat Rev Neurosci 2014;15:431-442

5. Byrne LC, Oztürk BE, Lee T, Fortuny C, Visel M, Dalkara D, Schaffer DV, Flannery JG. Retinoschisin gene therapy in photoreceptors, Müller glia or all retinal cells in the Rs1h-/- mouse. Gene Ther 2014;21:585-592

6. Surgucheva I, Shestopalov VI, Surguchov A. Effect of gamma-synuclein silencing on apoptotic pathways in retinal ganglion cells. J Biol Chem 2008;283:36377-36385

7. Sengillo JD, Justus S, Tsai YT, Cabral T, Tsang SH. Gene and cell-based therapies for inherited retinal disorders: an update. Am J Med Genet C Semin Med Genet 2016;172: 349-366

8. Jayaram H, Jones MF, Eastlake K, Cottrill PB, Becker S, Wiseman J, Khaw PT, Limb GA. Transplantation of photoreceptors derived from human Muller glia restore rod function in the P23H rat. Stem Cells Transl Med 2014;3:323-333

9. Angbohang A, Wu N, Charalambous T, Eastlake K, Lei Y, Kim YS, Sun XH, Limb GA. Downregulation of the canonical WNT signaling pathway by TGF $\beta 1$ inhibits photoreceptor differentiation of adult human müller glia with stem cell characteristics. Stem Cells Dev 2016;25:1-12

10. Park SC, Su D, Tello C. Anti-VEGF therapy for the treatment of glaucoma: a focus on ranibizumab and bevacizumab. Expert Opin Biol Ther 2012;12:1641-1647 
11. Horsley MB, Kahook MY. Anti-VEGF therapy for glaucoma. Curr Opin Ophthalmol 2010;21:112-117

12. Hu CJ, Wang LY, Chodosh LA, Keith B, Simon MC. Differential roles of hypoxia-inducible factor lalpha (HIF1alpha) and HIF-2alpha in hypoxic gene regulation. Mol Cell Biol 2003;23:9361-9374

13. Xin X, Rodrigues $M$, Umapathi $M$, Kashiwabuchi F, Ma T, Babapoor-Farrokhran S, Wang S, Hu J, Bhutto I, Welsbie DS, Duh EJ, Handa JT, Eberhart CG, Lutty G, Semenza GL, Montaner S, Sodhi A. Hypoxic retinal Muller cells promote vascular permeability by HIF-1-dependent up-regulation of angiopoietin-like 4. Proc Natl Acad Sci U S A 2013;110:E3425-E3434

14. Rodrigues $\mathrm{M}$, Xin X, Jee $\mathrm{K}$, Babapoor-Farrokhran S, Kashiwabuchi F, Ma T, Bhutto I, Hassan SJ, Daoud Y, Baranano D, Solomon S, Lutty G, Semenza GL, Montaner S, Sodhi A. VEGF secreted by hypoxic Müller cells induces MMP-2 expression and activity in endothelial cells to promote retinal neovascularization in proliferative diabetic retinopathy. Diabetes 2013;62:3863-3873

15. Liby K, Neltner B, Mohamet L, Burd C, Ben-Jonathan N. Endostatin expression by MDA-MB-435 breast cancer cells effectively inhibits tumor growth. Cancer Biol Ther 2003; 2:48-52

16. Faiq MA, Dada R, Qadri R, Dada T. CYP1B1-mediated pathobiology of primary congenital glaucoma. J Curr Glaucoma Pract 2015;9:77-80

17. Choudhary D, Jansson I, Schenkman JB. CYP1B1, a developmental gene with a potential role in glaucoma therapy. Xenobiotica 2009;39:606-615

18. Song WT, Zhang XY, Xia XB. Atoh7 promotes the differentiation of retinal stem cells derived from Müller cells into retinal ganglion cells by inhibiting Notch signaling. Stem Cell Res Ther 2013;4:94

19. Venturini C, Nag A, Hysi PG, Wang JJ, Wong TY, Healey PR, Mitchell P, Hammond CJ, Viswanathan AC; Wellcome Trust Case Control Consortium 2, BMES GWAS Group. Clarifying the role of ATOH7 in glaucoma endophenotypes. Br J Ophthalmol 2014;98:562-566

20. Yao J, Sun X, Wang Y, Xu G, Qian J. Math5 promotes retinal ganglion cell expression patterns in retinal progenitor cells. Mol Vis 2007;13:1066-1072

21. Song WT, Zeng Q, Xia XB, Xia K, Pan Q. Atoh7 promotes retinal Müller cell differentiation into retinal ganglion cells. Cytotechnology 2016;68:267-277

22. Hickmott JW, Chen CY, Arenillas DJ, Korecki AJ, Lam SL, Molday LL, Bonaguro RJ, Zhou M, Chou AY, Mathelier A, Boye SL, Hauswirth WW, Molday RS, Wasserman WW, Simpson EM. PAX6 MiniPromoters drive restricted expression from rAAV in the adult mouse retina. Mol Ther Methods Clin Dev 2016;3:16051

23. Maihöfner C, Schlötzer-Schrehardt U, Gühring H, Zeilhofer HU, Naumann GO, Pahl A, Mardin C, Tamm ER, Brune $\mathrm{K}$. Expression of cyclooxygenase- 1 and -2 in normal and glaucomatous human eyes. Invest Ophthalmol Vis Sci 2001;42:2616-2624
24. Barraza RA, McLaren JW, Poeschla EM. Prostaglandin pathway gene therapy for sustained reduction of intraocular pressure. Mol Ther 2010;18:491-501

25. Khare PD, Loewen N, Teo W, Barraza RA, Saenz DT, Johnson DH, Poeschla EM. Durable, safe, multi-gene lentiviral vector expression in feline trabecular meshwork. Mol Ther 2008;16:97-106

26. Ko ML, Hu DN, Ritch R, Sharma SC. The combined effect of brain-derived neurotrophic factor and a free radical scavenger in experimental glaucoma. Invest Ophthalmol Vis Sci 2000;41:2967-2971

27. Nafissi N, Foldvari $M$. Neuroprotective therapies in glaucoma: II. Genetic nanotechnology tools. Front Neurosci 2015;9:355

28. Kimura A, Namekata K, Guo X, Harada C, Harada T. Neuroprotection, growth factors and BDNF-TrkB signalling in retinal degeneration. Int J Mol Sci 2016;17:E1584 doi: 10.3390/ijms17091584

29. Hu Y, Cho S, Goldberg JL. Neurotrophic effect of a novel TrkB agonist on retinal ganglion cells. Invest Ophthalmol Vis Sci 2010;51:1747-1754

30. Johnson TV, Bull ND, Martin KR. Neurotrophic factor delivery as a protective treatment for glaucoma. Exp Eye Res 2011;93:196-203

31. Tassoni A, Gutteridge A, Barber AC, Osborne A, Martin KR. Molecular mechanisms mediating retinal reactive gliosis following bone marrow mesenchymal stem cell transplantation. Stem Cells 2015;33:3006-3016

32. Checa-Casalengua P, Jiang C, Bravo-Osuna I, Tucker BA, Molina-Martínez IT, Young MJ, Herrero-Vanrell R. Retinal ganglion cells survival in a glaucoma model by GDNF/Vit E PLGA microspheres prepared according to a novel microencapsulation procedure. J Control Release 2011;156: 92-100

33. Semba K, Namekata K, Kimura A, Harada C, Mitamura Y, Harada T. Brimonidine prevents neurodegeneration in a mouse model of normal tension glaucoma. Cell Death Dis 2014;5:e1341

34. Koeberle PD, Bähr M. The upregulation of GLAST-1 is an indirect antiapoptotic mechanism of GDNF and neurturin in the adult CNS. Cell Death Differ 2008;15:471-483

35. Ruberte J, Ayuso E, Navarro M, Carretero A, Nacher V, Haurigot V, George M, Llombart C, Casellas A, Costa C, Bosch A, Bosch F. Increased ocular levels of IGF-1 in transgenic mice lead to diabetes-like eye disease. J Clin Invest 2004;113:1149-1157

36. Ma J, Guo C, Guo C, Sun Y, Liao T, Beattie U, López FJ, Chen DF, Lashkari K. Transplantation of human neural progenitor cells expressing IGF-1 enhances retinal ganglion cell survival. PLoS One 2015;10:e125695

37. Zhao M, Andrieu-Soler C, Kowalczuk L, Paz Cortés M, Berdugo M, Dernigoghossian M, Halili F, Jeanny JC, Goldenberg B, Savoldelli M, El Sanharawi M, Naud MC, van Ijcken W, Pescini-Gobert $\mathrm{R}$, Martinet D, Maass A, Wijnholds J, Crisanti P, Rivolta C, Behar-Cohen F. A new CRB1 rat mutation links Müller glial cells to retinal 
telangiectasia. J Neurosci 2015;35:6093-6106

38. Alves CH, Pellissier LP, Vos RM, Garcia Garrido M, Sothilingam V, Seide C, Beck SC, Klooster J, Furukawa T, Flannery JG, Verhaagen J, Seeliger MW, Wijnholds J. Targeted ablation of Crb2 in photoreceptor cells induces retinitis pigmentosa. Hum Mol Genet 2014;23:3384-3401

39. Pellissier LP, Alves CH, Quinn PM, Vos RM, Tanimoto N, Lundvig DM, Dudok JJ, Hooibrink B, Richard F, Beck SC, Huber G, Sothilingam V, Garcia Garrido M, Le Bivic A, Seeliger MW, Wijnholds J. Targeted ablation of CRB1 and CRB2 in retinal progenitor cells mimics Leber congenital amaurosis. PLoS Genet 2013;9:e1003976

40. Dallaire A, Simard MJ. The implication of microRNAs and endo-siRNAs in animal germline and early development. Dev Biol 2016;416:18-25

41. Yu-Wai-Man C, Tagalakis AD, Manunta MD, Hart SL, Khaw PT. Receptor-targeted liposome-peptide-siRNA nanoparticles represent an efficient delivery system for MRTF silencing in conjunctival fibrosis. Sci Rep 2016;6:21881

42. Mahdy MA. Gene therapy in glaucoma-3: Therapeutic approaches. Oman J Ophthalmol 2010;3:109-116

43. Chiasseu M, Cueva Vargas JL, Destroismaisons L, Vande Velde C, Leclerc N, Di Polo A. Tau accumulation, altered phosphorylation, and missorting promote neurodegeneration in glaucoma. J Neurosci 2016;36:5785-5798

44. Webber HC, Bermudez JY, Sethi A, Clark AF, Mao W. Crosstalk between TGF $\beta$ and Wnt signaling pathways in the human trabecular meshwork. Exp Eye Res 2016;148: 97-102

45. Gonzalez JM Jr. Existence of the canonical Wnt signaling pathway in the human trabecular meshwork. Invest Ophthalmol Vis Sci 2012;53:6972

46. Brennan LA, Kantorow M. Mitochondrial function and redox control in the aging eye: role of MsrA and other repair systems in cataract and macular degenerations. Exp Eye Res 2009;88:195-203

47. Saccà SC, Roszkowska AM, Izzotti A. Environmental light and endogenous antioxidants as the main determinants of non-cancer ocular diseases. Mutat Res 2013;752:153-171

48. Wu JH, Zhang SH, Nickerson JM, Gao FJ, Sun Z, Chen XY, Zhang SJ, Zhang R, Gao F, Chen JY, Luo Y, Wang $\mathrm{Y}$, Sun XH. Cumulative mtDNA damage and mutations contribute to the progressive loss of RGCs in a rat model of glaucoma. Neurobiol Dis 2015;74:167-179

49. Jiang W, Tang L, Zeng J, Chen B. Adeno-associated virus mediated SOD gene therapy protects the retinal ganglion cells from chronic intraocular pressure elevation induced injury via attenuating oxidative stress and improving mitochondrial dysfunction in a rat model. Am J Transl Res 2016;8:799-810

50. Kim KY, Perkins GA, Shim MS, Bushong E, Alcasid N, Ju S, Ellisman MH, Weinreb RN, Ju WK. DRP1 inhibition rescues retinal ganglion cells and their axons by preserving mitochondrial integrity in a mouse model of glaucoma. Cell Death Dis 2015;6:e1839

51. Petit L, Punzo C. Gene therapy approaches for the treat- ment of retinal disorders. Discov Med 2016;22:221-229

52. Cotrim AP, Baum BJ. Gene therapy: some history, applications, problems, and prospects. Toxicol Pathol 2008;36: 97-103

53. Joseph M, Trinh HM, Cholkar K, Pal D, Mitra AK. Recent perspectives on the delivery of biologics to back of the eye. Expert Opin Drug Deliv 2017;14:631-645

54. Murphy N, Lynch K, Lohan P, Treacy O, Ritter T. Mesenchymal stem cell therapy to promote corneal allograft survival: current status and pathway to clinical translation. Curr Opin Organ Transplant 2016;21:559-567

55. Clements LE, Garvican ER, Dudhia J, Smith RK. Modulation of mesenchymal stem cell genotype and phenotype by extracellular matrix proteins. Connect Tissue Res 2016;57: 443-453

56. Harper MM, Grozdanic SD, Blits B, Kuehn MH, Zamzow D, Buss JE, Kardon RH, Sakaguchi DS. Transplantation of BDNF-secreting mesenchymal stem cells provides neuroprotection in chronically hypertensive rat eyes. Invest Ophthalmol Vis Sci 2011;52:4506-4515

57. Wang Y, Ying Y, Cui X. Effects on proliferation and differentiation of human umbilical cord-derived mesenchymal stem cells engineered to express neurotrophic factors. Stem Cells Int 2016 doi: 10.1155/2016/1801340

58. Kode JA, Mukherjee S, Joglekar MV, Hardikar AA. Mesenchymal stem cells: immunobiology and role in immunomodulation and tissue regeneration. Cytotherapy 2009; 11:377-391

59. Johnson TV, Bull ND, Hunt DP, Marina N, Tomarev SI, Martin KR. Neuroprotective effects of intravitreal mesenchymal stem cell transplantation in experimental glaucoma. Invest Ophthalmol Vis Sci 2010;51:2051-2059

60. Nadri S, Yazdani S, Arefian E, Gohari Z, Eslaminejad MB, Kazemi B, Soleimani M. Mesenchymal stem cells from trabecular meshwork become photoreceptor-like cells on amniotic membrane. Neurosci Lett 2013;541:43-48

61. Yu S, Tanabe T, Dezawa M, Ishikawa H, Yoshimura N. Effects of bone marrow stromal cell injection in an experimental glaucoma model. Biochem Biophys Res Commun 2006;344:1071-1079

62. Wilkins A, Kemp K, Ginty M, Hares K, Mallam E, Scolding $\mathrm{N}$. Human bone marrow-derived mesenchymal stem cells secrete brain-derived neurotrophic factor which promotes neuronal survival in vitro. Stem Cell Res 2009;3:63-70

63. Weiss JN, Levy S, Malkin A. Stem Cell Ophthalmology Treatment Study (SCOTS) for retinal and optic nerve diseases: a preliminary report. Neural Regen Res 2015;10: 982-988

64. Cocks G, Curran S, Gami P, Uwanogho D, Jeffries AR, Kathuria A, Lucchesi W, Wood V, Dixon R, Ogilvie C, Steckler T, Price J. The utility of patient specific induced pluripotent stem cells for the modelling of Autistic Spectrum Disorders. Psychopharmacology (Berl) 2014;231: 1079-1088

65. Kang E, Wang X, Tippner-Hedges R, Ma H, Folmes CD, Gutierrez NM, Lee Y, Van Dyken C, Ahmed R, Li Y, Koski 
A, Hayama T, Luo S, Harding CO, Amato P, Jensen J, Battaglia D, Lee D, Wu D, Terzic A, Wolf DP, Huang T, Mitalipov S. Age-related accumulation of somatic mitochondrial DNA mutations in adult-derived human iPSCs. Cell Stem Cell 2016;18:625-636

66. Gundry MC, Brunetti L, Lin A, Mayle AE, Kitano A, Wagner D, Hsu JI, Hoegenauer KA, Rooney CM, Goodell MA, Nakada D. Highly Efficient genome editing of murine and human hematopoietic progenitor cells by CRISPR/
Cas9. Cell Rep 2016;17:1453-1461

67. Hu X. CRISPR/Cas9 system and its applications in human hematopoietic cells. Blood Cells Mol Dis 2016;62:6-12

68. Zhu W, Gramlich OW, Laboissonniere L, Jain A, Sheffield VC, Trimarchi JM, Tucker BA, Kuehn MH. Transplantation of iPSC-derived TM cells rescues glaucoma phenotypes in vivo. Proc Natl Acad Sci U S A 2016;113:E3492E3500 